\title{
Dificultades en el factor neuropsicológico Cinestésico predicen posibles problemas en la adquisición de la escritura*
}

Kinesthetic neuropsychological factor difficulties predict possible problems in writing acquisition

DOI: 10.11144/Javeriana.upsy15-5.dfnc
Recepción: 05 Septiembre 2016 | Aprobación: 16 Noviembre 2016

\author{
María Jimena Sarmiento-Bolaños ${ }^{\mathrm{a}}$ \\ Universidade Federal do Paraná, Brasil \\ Inés Adriana Rojas Ríos \\ Instituto Colombiano de Neurociencias, Colombia \\ María Jeimy Moreno Carrillo \\ Universidad de San Buenaventura, Colombia
}

Alexander Gómez-A

Universidade Federal do Paraná, Brasil

aE-mail: mariajimenasarmiento@gmail.com

Para citar este artículo: Sarmiento-Bolaños, M. J., Rojas, I. A., Moreno, C. M. J., \& Gómez-A, A. (2016). Dificultades en el factor neuropsicológico Cinestésico predicen posibles problemas en la adquisición de la escritura. Universitas Psychologica, 15(5). http:// dx.doi.org/10.11144/Javeriana.upsy15-5.dfnc

\section{RESUMEN}

En este estudio, se evaluó a un grupo de niños y niñas de primero a tercer grado de educación básica primaria con problemas en la adquisición de la escritura en comparación con un grupo control. Los resultados obtenidos en la Evaluación neuropsicológica infantil "PueblaSevilla”, muestran diferencias significativas en los factores Cinético, Cinestésico y Percepción Espacial Global entre los dos grupos. Resultados similares fueron observados en las tareas de escritura contenidas en la Evaluación Neuropsicológica Infantil (ENI). Asimismo, se tipificaron cualitativamente los tipos de errores cometidos en las tareas de escritura. Análisis posteriores mostraron una correlación en la ejecución de los sujetos, mostraron además que el factor Cinestésico puede considerarse predictor de una adecuada adquisición de la escritura y que la tarea de dictado de la ENI es sensible para el reconocimiento de dificultades en dicho factor.

Palabras clave

análisis cinestésico; proceso de lecto-escritura; neuropsicología.

\section{ABSTRACT}

In this study, we evaluated a group of boys and girls, from first to third grade of primary school, with difficulties in writing acquisition in comparison with a control group. The results obtained by using the "Evaluación Neuropsicológica infantil 'Puebla-Sevilla" show significant differences between the experimental and the control group in the Kinetic, Kinesthesic and the Global Spatial Perception neuropsychological factors. Similar results were also obtained in the writing tasks that are part of the "Evaluación Neuropsicológica Infantil (ENI)". We also qualitatively typified the errors in the writing tasks. Correlational statistical analysis of the experimental and control groups show that the Kinesthesic factor can predict possible difficulties in the correct acquisition of the writing, and also, that the dictation task of the ENI can be valid for the recognition of difficulties in that factor. 
María Jimena Sarmiento-Bolaños, Inés Adriana Rojas Ríos, María Jeimy Moreno Carrillo, Alexander Gómez-A.

Keywords

kinesthesic analysis; reading-writing process; neuropsychology.

\section{Introducción}

Los problemas del aprendizaje, particularmente aquellos relacionados con procesos complejos como la adquisición de la escritura en la edad escolar, ocupan un lugar importante en la agenda de educadores, padres y terapeutas. Las aproximaciones al estudio de estas problemáticas han sido diversas y cada una expone lo que dentro de su cuerpo teórico-metodológico considera más relevante (Eslava-Cobos \& Mejía, 2008; Yañez, 2008). En el caso de la escuela neuropsicológica de enfoque Histórico-Cultural (herencia de L.S. Vigotsky y A. R. Luria, principalmente), la aproximación hacia los problemas del aprendizaje escolar se realiza a través del análisis cuidadoso de las acciones escolares. Esta escuela considera las funciones psicológicas como sistemas funcionales complejos dentro de los cuales, varias de éstas funciones pueden aparecer en dependencia de la actividad propia del sujeto, todo esto, siguiendo la premisa de la localización sistémica y dinámica de las funciones psicológicas (Luria, 1969; Vigotsky, 1979). De esta manera, el objeto de estudio de la neuropsicología no son las funciones psicológicas "per se", sino más bien, la conformación de dichos sistemas bajo diversas condiciones de desarrollo (Quintanar \& Solovieva, 2002). Para tal fin, surge el término factor o mecanismo neuropsicológico, cuyo papel es mediar entre la actividad propia del cerebro y la función (o funciones) psicológica(s), ya que un mismo factor puede participar en la conformación de una o varias funciones diferentes. De este modo, la evaluación, diagnóstico y futura corrección, tienen como finalidad establecer el mecanismo o mecanismos responsables por las fallas en una $\mathrm{u}$ otra función o actividad psicológica (Quintanar \& Solovieva, 2002).

Luria (1950) describió algunos de los componentes del sistema funcional complejo de la escritura. Entre estos, se encuentran, como componentes de tipo posterior (es decir, asociados a zonas occipitales, temporales y parietales), el análisis auditivo (factor auditivo), el análisis cinestésico (factor cinestésico), la organización visual (factor visual) y la organización visoespacial (factores espacial, analítico y global) de la escritura. Como componentes anteriores (asociados a zonas frontales) se encuentran la organización cinética de los movimientos gráficos y del programa motor (factor cinético) y la idea de la comunicación escrita y de su realización (factor de regulación y control). De acuerdo con Akhutina (2002), a esta descripción inicial, habría que sumarle dos componentes claves para el proceso: la participación del primer bloque funcional ("energético"), que se relaciona con estructuras profundas y del tronco cerebral que participan en la regulación del tono cortical y la diferenciación hemisférica, en especial del hemisferio derecho, como aporte a todo el sistema (ver tabla Anexo 1, material suplementario).

Entre todos estos componentes el factor o mecanismo cinestésico ocupa un lugar destacado para la presente investigación. Dicho mecanismo hace parte de los denominados factores modalespecíficos (Xomskaya, 2002), que se relacionan con el trabajo de sistemas de analizadores específicos (visual, auditivo, motor y cinestésicotáctil). La alteración del factor cinestésico puede evidenciar diversos defectos gnósicos (agnosia táctil), diferentes formas de apraxias y alteraciones sensoriales del lenguaje, así como variados tipos de alteraciones mnésicas (v.g. alteración de memoria táctil) (Xomskaya, 2002). El desarrollo de este factor se inicia en etapas tempranas del desarrollo, entre uno y dos años de edad, etapa en la cual maduran los sectores parietales que garantizan la percepción cinesésico-tactil, seguido por el desarrollo de sectores temporales y occipitales (Quintanar \& Solovieva, 2005).

En función de lo anterior, la principal diferencia entre el abordaje Luriano y otros abordajes para los problemas durante el aprendizaje, radica en el hecho que, a diferencia de la identificación de los síntomas normalmente asociados al término disgrafia, el análisis del sistema funcional permite establecer los mecanismos o factores directamente implicados 
en las fallas de la actividad en la escritura (Akhutina, 2002; Rojas \& Solovieva, 2005). De esta forma, para un mismo "síntoma" recurrente en las fallas de escritura, como la sustitución de letras en una misma palabra (por ejemplo sustituciones como losa por rosa, día por tía y caza por casa), el análisis factorial establecería hasta tres posibles alteraciones diferentes: alteraciones del mecanismo cinestésico, alteraciones del mecanismo fonemático, o alteraciones del mecanismo de regulación y control, respectivamente. El establecimiento de uno u otro mecanismo como responsable de las dificultades observadas, orienta el programa de prevención y corrección a seguir. Adicionalmente, entendiendo la lógica de la alteración sistémica, podrían ser intervenidas posibles alteraciones en otros sistemas funcionales que incluyan al mecanismo identificado en su estructura.

De otro lado, estudios realizados en países de habla hispana sobre dificultades de escritura y factores neuropsicológicos asociados, han identificado la alta prevalencia de dificultades en el aprendizaje, (observada, por ejemplo, en la práctica clínica) y la necesidad de intervenir sobre estos. Entre ellos se encuentra uno realizado en Perú (Canales, Velarde, Meléndez \& Lingán, 2013), algunos realizados en México (Cisneros, 2009; Mata, Solovieva, Quintanar $\&$ Soto, 2014) y uno reciente en Colombia acerca de la tipificación de los errores de lectura y escritura en niños de $2^{\circ}$ grado (Londoño, Jiménez, González \& Solovieva, 2016). Sin embargo, según Bravo-Valdivieso, Cuadros, Mejía y Eslava (2015), en Latinoamérica y particularmente en Colombia, los estudios, en su mayoría, plantean la prevalencia, descripción y tipificación de los trastornos escolares y de aprendizaje ligados a factores socio-económicos y emocionales-familiares, la descripción de los síndromes, su comorbilidad con el Trastorno con Déficit de Atención (TDA) y la efectividad de la medicación, pero solo en los últimos años se explora la relación entre la neuropsicología y los trastornos del aprendizaje. Sin embargo y de forma paradójica, es posible encontrar estudios que evalúan la efectividad de diferentes programas para remediar las dificultades en la lecto-escritura (por ejemplo, González, Martín \& Delgado, 2011) sin que haya una identificación previa de las causas que subyacen a dichas dificultades. Asimismo, García, Madrazo y Viñal (2002), clasifican las ejecuciones de escritura en tres tipos de alteración: disgrafía superficial, (dificultad para las palabras ortográficamente arbitrarias e irregulares), disgrafía fonológica, (dificultad para convertir fonemas en grafemas) y disgrafía profunda, (dada por la concurrencia de los trastornos anteriores en un nivel severo), aunque las causas de la dificultad en el nivel neuropsicológico no fueron identificadas.

Teniendo en cuenta los antecedentes, el presente estudio surge de la necesidad de profundizar en las posibles causas que subyacen a las dificultades de escritura de niños entre primero y tercer año de primaria, y específicamente, en aquellas relacionadas con los diferentes mecanismosfactores neuropsicológicos.

\section{Método}

El presente fue un estudio de tipo descriptivocorrelacional. Las características generales de la muestra, la batería de evaluación y el procedimiento son descritos a continuación.

\section{Participantes}

En el presente estudio participaron un total de 59 alumnos de los grados primero (1RO), segundo (2DO) y tercero (3RO) de básica primaria de una institución educativa privada, con edades entre los 7 y los 10 años. Entre los participantes, veinticuatro (24) fueron niñas y treinta y cinco (35) fueron niños. De la muestra total, 39 sujetos fueron asignados al grupo de problemas en la escritura (PE) y los restantes al grupo control (CT). Dicha distribución se realizó con base en el reporte del cuerpo docente y el seguimiento que este hizo de cada alumno durante el periodo académico. En términos generales, el cuerpo docente baso la selección de los casos con problemas de escritura en el 
análisis de las tareas en casa y en el aula de clase, dictados y evaluaciones durante el año escolar. Especial importancia fue dada a los reportes desde áreas relacionadas con la escritura como español, geografía y matemáticas (entre otras). La información derivada del seguimiento a cada alumno fue socializada en las reuniones entre docentes de los cursos incluidos en el estudio y a partir de ella, los casos con mayor visibilidad fueron seleccionados. Dado que la idea de la presente investigación no era clasificar las dificultades en la escritura de acuerdo con los posibles factores que podrían estarlas generando, todos los participantes que fueron identificados por los docentes con problemas en la escritura fueron incluidos. Los criterios de inclusión y exclusión que fueron tenidos en cuenta para la selección de la muestra están contenidos en la Tabla Anexo 2. De igual manera, las características generales de la muestra pueden ser observadas en la Tabla Anexo 3.

\section{Instrumentos}

La evaluación neuropsicológica de los participantes se realizó a través de la prueba Evaluación neuropsicológica infantil "PueblaSevilla” (ENIP-S) - Screening Neuropsicológico Clínico (Solovieva, Quintanar \& León-Carrión, 2007), la cual cuenta con una consistencia interna de $\#=0.93$, alta consistencia intraescalas y general, encontrándose en el análisis factorial una aglomeración para siete de los factores propuestos por el enfoque históricocultural, así como una validez convergente con pruebas de dominio específico (Rivera et al., 2011). El protocolo está conformado por diez apartados, cada uno de los cuales incluye diez ítems. Las tareas del instrumento caracterizan el estado funcional de los factores neuropsicológicos: Programación y control (PyC), Organización secuencial motora-cinético (CIN), Integración cinestésica táctil-cinestésico (CNT), Evaluación de la discriminación verbalauditiva-oído fonemático (OF), Retención audio-verbal (RA-V) y visual (RV), Percepción espacial Global (PEG), Percepción espacial analítica (PEA) y Atención y estado de alerta (At). La ENIP-S ha sido caracterizada en población mexicana (Solovieva, Loredo, Quintanar \& Lázaro, 2013) y empleada en la práctica clínica para el diagnóstico y corrección de trastornos del desarrollo en niños. Dada la necesidad de evaluar el estado actual de los diferentes factores neuropsicológicos en los participantes así como el hecho que esta es la única prueba que, además de determinar dicho estado, es derivada directamente del modelo Histórico-Cultural, su selección fue evidente. De otro lado, fueron empleadas las sub-pruebas de Habilidades Metalingüísticas y Escritura contenidas en la Evaluación Neuropsicológica Infantil (ENI), la cual fue validada para población colombiana por Rosselli et al., (2004). Brevemente, la primera sub-prueba de la ENI está compuesta por cuatro tareas y la segunda por siete (habilidades metalingüísticas, escritura del nombre, dictado de sílabas, dictado de palabras, dictado de no palabras, dictado de oraciones, copia de un texto y recuperación escrita). Estas subpruebas fueron seleccionadas para ser aplicadas en el marco de la presente investigación dada su especificidad en la evaluación de la escritura (para una revisión más en detalle puede remitirse a Roselli et al., 2004).

\section{Procedimiento}

Para la obtención de la muestra se estableció un contacto inicial con la institución educativa con el fin de explicar los objetivos y alcances de la investigación. Posteriormente fueron seleccionados los participantes a través del reporte realizado por los profesores de cada grado escolar acerca de los alumnos que presentaban problemas en la escritura. Una vez identificados los potenciales participantes, se realizó una reunión con los padres de familia en la cual fueron informados acerca de la investigación y sus objetivos y allí mismo fue solicitada la autorización que permitiese a sus hijos participar del estudio. Cuando el grupo final de participantes fue claro, un equipo de profesionales entrenados (neuropsicólogos 
clínicos) llevó a cabo el proceso de evaluación en las instalaciones del colegio. El tiempo total de aplicación fue de aproximadamente una hora y media siendo aplicada la ENIP primero y posteriormente las subpruebas de la ENI. La evaluación se realizó de manera individual en espacios diferentes al aula de clase. Posteriormente, se realizó el análisis estadístico de los datos recogidos y las interpretaciones derivadas de los mismos. Finalizado el proceso, los resultados y conclusiones del estudio fueron socializados con la institución educativa.

\section{Análisis de datos}

La comparación de los puntajes en la ENIP$S$ entre cursos y edades fue realizada usando ANOVA de una vía seguida de test post-hoc de Bonferroni. Las comparaciones restantes entre los grupos CT y PE, fueron realizadas usando la prueba $\mathrm{t}$-Student. El nivel de significancia para todos los análisis estadísticos fue $\mathrm{p}<0.05$. Finalmente, el procesamiento de los datos fue conducido usando el paquete estadístico Prism para Windows, versión 5.03.

\section{Resultados}

Los análisis estadísticos iniciales fueron realizados tomando como base los resultados obtenidos por los participantes en la ENIP-S. En la primera comparación, fueron incluidos los datos de todos los participantes asignados a cada grupo (CT y PE) independientemente del grado escolar o la edad. Este análisis mostró que los resultados del grupo control (CT) fueron significativamente mayores en comparación con los obtenidos por el grupo con problemas en la escritura $(\mathrm{PE})(\mathrm{t}=3.36, \mathrm{p}<0.001$; Figura 1). Posteriormente, y dadas las diferencias de edad y grado escolar de los participantes, se llevaron a cabo comparaciones por separado para cada una de esas variables entre los grupos CT y PE. Debido que la muestra fue "no probabilística" y que la participación de los sujetos debía ser autorizada por sus padres, la conformación final de los grupos control y experimental estuvo sujeta a dicha situación (ver tabla anexo 2). Lo anterior llevó a tener un número dispar de sujetos control para cada curso y grupo de edad, algo que fue particularmente evidente en el grado 2DO, donde solo dos participantes pudieron ser asignados al grupo CT. Debido a esto, se decidió comparar los resultados de los sujetos CT por edad y grado escolar explorando la posibilidad de unir grupos en aquellos casos donde no fuesen halladas diferencias significativas. La comparación de los sujetos CT por edad mostró ser significativa ( $F$ $=4.87, \mathrm{p}=0.014)$. Sin embargo, el análisis pos-hoc (Test de Bonferroni) arrojó diferencias entre los sujetos de 7 y 9 años $(\mathrm{p}<0.05$; Figura 1), pero no entre los grupos por edad restantes. A continuación, se llevaron a cabo las comparaciones del grupo control por grado escolar, hallándose diferencias significativas entre los puntajes de los diferentes grupos $(\mathrm{F}=9.23$, $\mathrm{p}$ $=0.001$; Figura 1). Interesantemente, el análisis pos-hoc mostró diferencias solo entre los grupos $1 \mathrm{RO}$ y $3 \mathrm{RO}(\mathrm{p}<0.01)$, pero no entre $1 \mathrm{RO}$ y 2DO o 2DO y $3 \mathrm{RO}$. Este resultado colocó a los sujetos del grado $2 \mathrm{DO}$ en un punto intermedio entre $1 \mathrm{RO}$ y $3 \mathrm{RO}$, donde sus resultados no fueron diferentes de los obtenidos por los participantes de los otros dos cursos. Para determinar si esta tendencia era coherente entre las edades, se llevó a cabo el mismo análisis por grado escolar para el grupo PE. De manera consistente, fue encontrada la misma tendencia que en el grupo control, identificándose una diferencia significativa por grado escolar $(\mathrm{F}=3.71, \mathrm{p}=0.034$, Figura 1$)$. El análisis pos-hoc mostró que solo los grupos de $1 \mathrm{RO}$ y $3 \mathrm{RO}$ fueron diferentes y que el grupo 2DO no tenía diferencias con $1 \mathrm{RO}$ o $3 \mathrm{RO}$. Basados en este análisis, se decidió que el grupo control para el grado 2DO se constituiría de la suma de todos los sujetos control de la muestra, dado que no fueron halladas diferencias a través de los análisis estadísticos previos. 
María Jimena Sarmiento-Bolaños, Inés Adriana Rojas Ríos, María Jeimy Moreno Carrillo, Alexander Gómez-A.

\section{Figura 1}

Comparación de los resultados por grupos
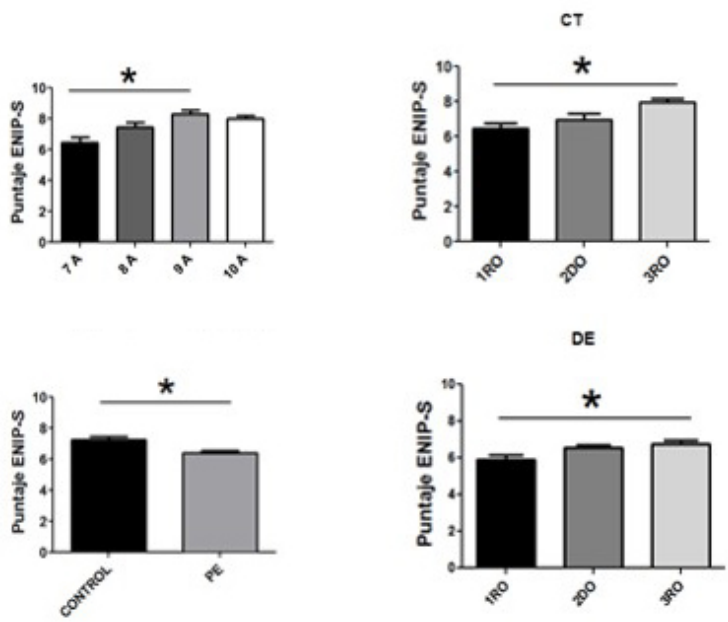

Comparación de los resultados en la ENIP-S por grupo (A), edad (B) y grado escolar (C y D). Las comparaciones por grado escolar entre los sujetos de cada grupo (CT y $\mathrm{PE}$ ) no mostró diferencias de los sujetos del grupo $2 \mathrm{DO}$ con ningún otro de los cursos ( $\mathrm{p}>0$-05). A partir de este análisis, el grupo control para ese grado escolar (2DO) fue el total de participantes control del estudio. Fuente: elaboración propia.

Diferencias entre grupos por Factor Neuropsicológico (ENIP-S)

El siguiente análisis fue dirigido a identificar diferencias entre los participantes para cada uno de los factores neuropsicológicos evaluados a través de la ENIP-S. Este análisis se llevó a cabo tomando los puntajes obtenidos en los ítems que evalúan cada factor y comparando el grupo CT con el grupo PE en los tres grados escolares. La primera comparación fue realizada entre el grupo control y el experimental como un todo, sin diferenciar entre los grados escolares (grupo control $n=20$, grupo PE $n$ = 39). Los resultados evidenciaron diferencias para los factores Cinético $(\mathrm{t}=2.32, \mathrm{p}=0.024)$, Cinestésico $(t=2.44, p=0.018)$ y Percepción espacial global $(t=2.22, p=0.03$; Figura 2$)$. Los factores restantes no mostraron diferencias estadísticamente significativas, aunque existe una clara tendencia en el grupo PE a presentar puntajes más bajos en algunos factores, en comparación con el grupo control (v.g. retención audio-verbal, Figura 2).

Figura 2

Diferencias generales y por grado escolar entre los grupos CT y PE para cada Factor Neuropsicológico.
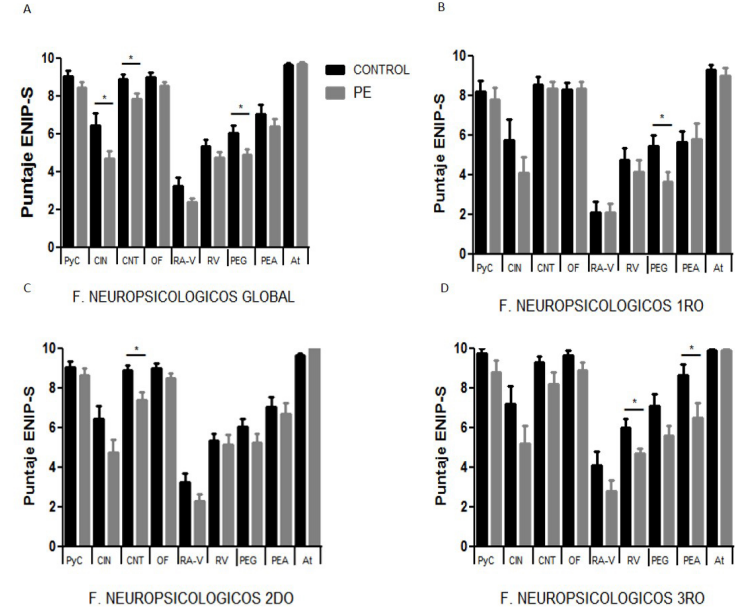

A. Comparación en los puntajes en la ENIP. S entre el grupo CT $(n=20)$ y el grupo PE $(n=39)$. B, C, D. Comparación de puntajes en la ENIP-S entre grupo CT y grupo PE por grado escolar. El nivel de significancia para todas las comparaciones fue de $\mathrm{p}<0.05$.

Fuente: elaboración propia.

Un análisis posterior fue realizado, tomando los puntajes por grado escolar y comparándolos con su respectivo grupo control. Para el grupo 1RO fueron encontradas diferencias significativas solo en los puntajes correspondientes al factor PEG $(\mathrm{t}=2.35$, $\mathrm{p}=0.03$; Figura 2). Los resultados también muestran una diferencia importante, aunque no significativa $(\mathrm{p}>0.05)$ en los puntajes del factor Cinético entre los dos grupos.

En el grado 2DO se hallaron diferencias significativas en el factor Cinestésico $(t=3.16$, $\mathrm{p}=0.003$; Figura 2) pero no en los restantes, sin embargo y de forma similar al grado $1 \mathrm{RO}$, no solo existe una marcada tendencia a la baja en los resultados del grupo PE en comparación con los controles, sino que, además, dicha tendencia es nuevamente evidente en el factor cinético (figura 2), lo que da sentido al resultado global (figura 2) donde fue observado un patrón similar. Finalmente, el grado $3 \mathrm{RO}$ mostró diferencias 
significativas en dos factores, $\mathrm{RV}(\mathrm{t}=2.60, \mathrm{p}=$ $0.018)$ y PEA $(t=2.22, p=0.04$; Figura 2$)$. Los restantes factores no mostraron diferencias estadísticamente significativas, aunque en $3 \mathrm{RO}$ también se mantiene la tendencia a la baja del grupo PE en el factor Cinético, sumándose además el factor RV.

\section{Diferencias entre grupos por Tipo de Error (ENI)}

A continuación, fue comparada la ejecución de los participantes en las pruebas específicas de la Evaluación Neuropsicológica Infantil (ENI). Los resultados no señalaron diferencias significativas para el grado $1 \mathrm{RO}$ en ninguna de las comparaciones realizadas, mientras que para el grado 2DO fue encontrada una diferencia significativa en Errores de Dictado $(\mathrm{t}=3.08$, $p=0.004$; Figura 3). En relación con el grado 3RO, se observaron diferencias significativas para los Errores de Dictado $(t=3.34, p=0.005$; Figura 3). Para los dos tipos de error restantes, fue observada una diferencia marginal tanto en los errores de copia $(\mathrm{t}=2.13, \mathrm{p}=0.055 \#)$ como de recuperación $(t=2.06, p=0.056 \#$; Figura 3). Es de resaltar que los sujetos $\mathrm{PE}$ del grado escolar 1RO, no fueron diferentes de sus controles, mientras que en los grados 2DO y $3 \mathrm{RO}$ fueron evidenciadas diferencias en uno (Errores dictado-2DO) o más tipos de error (E. dictado, copia y recuperación-3RO), mostrando una tendencia al aumento en la medida que el grado escolar fue mayor.

\section{Correlación entre Factores Neuropsicológicos (ENIP-S) y tipo de error (ENI)}

El siguiente paso fue establecer si existía alguna relación entre los factores neuropsicológicos que puntuaron bajo en el grupo PE para cada grado escolar y los diferentes tipos de error evaluados por la ENI. Surgió entonces la pregunta para cada uno de los grados escolares, iexiste alguna relación entre los puntajes bajos en los factores neuropsicológicos obtenidos por el grupo CT vs el grupo PE y el número de errores en la ENI? Para resolver dicha pregunta se realizaron tres análisis diferentes, el primero de los cuales incluyo la totalidad de los datos de la ENIP-S contrastados con el total de los datos para cada uno de los errores evaluados a través de la ENI. Ninguno de los análisis evidenció relación alguna entre los datos (datos no mostrados).

\section{Figura 3}

Diferencias entre grupos por Tipo de Error (ENI)

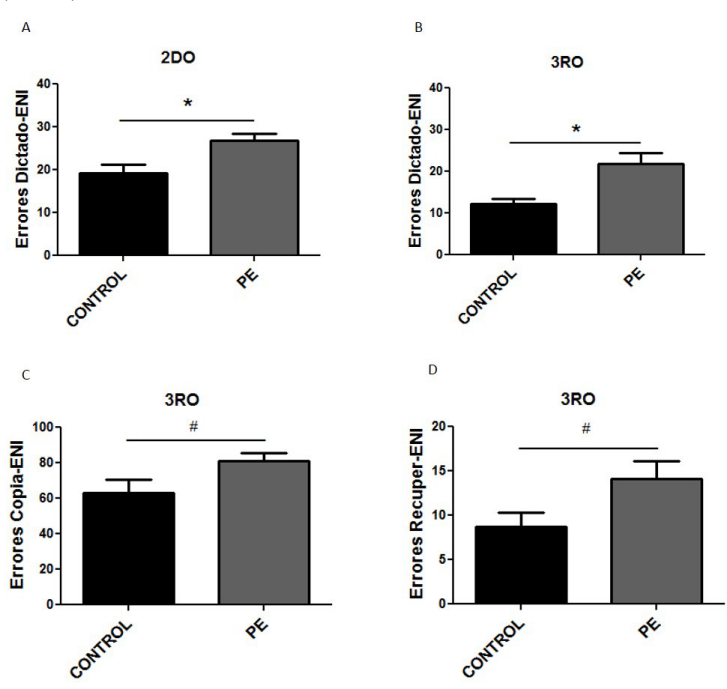

A. Errores en dictado - 2DO. B. Errores en dictado - 3RO. C. Errores en copia - 3RO. D. Errores en recuperación - 3RO. Las comparaciones restantes no fueron significativas. El nivel de significancia para todas las comparaciones fue de $\mathrm{p}<0.05$. Fuente: elaboración propia.

En segundo lugar, fueron comparados el total de los datos de la ENIP-S contra los datos obtenidos para cada uno de los errores de manera independiente en el grupo CT y en el grupo PE. Los resultados mostraron correlaciones negativas estadísticamente significativas para la prueba de Errores en Dictado-grupo CT( $\mathrm{r}=$ -0.520, $\mathrm{p}=0.018)$ y grupo PE $(\mathrm{r}=-0.392, \mathrm{p}$ $=0.013$; Figura 4). Para los otros errores, no fue encontrada correlación alguna $(\mathrm{p}>0.05$, datos no mostrados). Estos resultados fueron acordes con las predicciones realizadas cuando el estudio fue planificado, en términos que, a mayores puntajes en la ENIP-S se esperaría un menor número de errores en la prueba de la ENI, particularmente en dictado, donde el participante no cuenta con un modelo a seguir. 
María Jimena Sarmiento-Bolaños, Inés Adriana Rojas Ríos, María Jeimy Moreno Carrillo, Alexander Gómez-A.

Para el siguiente análisis, fueron contrastados los puntajes de aquellos factores donde se encontraron diferencias significativas entre el grupo CT y el PE, reportados en la parte inicial de estos resultados (Fig. 3). Dicho análisis incluyó tomar los datos de los diferentes factores en cada grupo escolar y contrastarlos con los valores en cada uno de los errores, en cada grado escolar y para cada grupo (CT y PE). Como no hubo diferencias en el grado escolar $1 \mathrm{RO}$, no fueron realizados análisis para ese grupo. Para el grado 2DO, fueron tomados los datos del Factor Cinestésico (Figura 2) y contrastados con los datos de cada error en el grupo CT y PE. No se encontraron correlaciones significativas en este análisis. Finalmente, para el grado escolar 3RO fueron tomados los factores RV y PEA.

\section{Figura 4}

Correlación entre Factores Neuropsicológicos y tipo de error
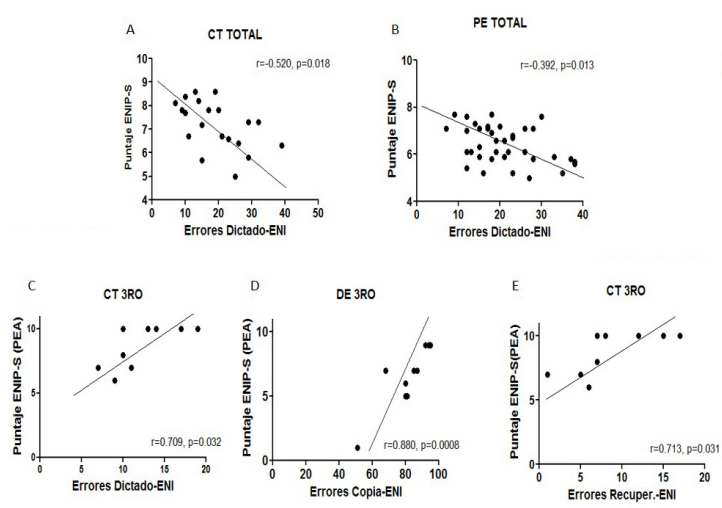

A. y B. Resultados del análisis correlacional para errores de dictado en el grupo total de sujetos del grupo CT y el grupo PE. El análisis correlacional del grado escolar 3RO para errores de dictado (C), errores de copia (D) y errores de recuperación $(\mathrm{E})$. Los valores de r y p son mostrados en cada gráfica.

El nivel de confianza utilizado fue de $\mathrm{p}<0.05$.

Fuente: elaboración propia.

El análisis del factor RV y los diferentes tipos de error no arrojaron ninguna correlación significativa. Sin embargo, cuando el factor PEA fue comparado con cada uno de los errores, fueron encontradas correlaciones significativas en errores de dictado para el grupo CT $(\mathrm{r}=$ 0.709, $\mathrm{p}=0.032$ ), Errores de Copia para el grupo PE $(r=0.88, p<0.001)$ y Errores en Recuperación para el grupo CT $(r=0.713, p$
$=0.031 ;$ Figura 4). Dichas correlaciones fueron positivas, algo contrario a lo que las predicciones y los resultados del análisis anterior habrían permitido esperar, dado que mayores puntajes en la ENIP-S permitirían predecir un menor número de errores en las subpruebas de la ENI y no lo contrario. La correlación positiva mencionada, se explica por la "no especificidad de los errores" con respecto al factor con el cual se contrasta. El siguiente paso fue analizar los tipos de error en las modalidades de dictado, copia y recuperación, de acuerdo con el factor neuropsicológico con el que podrían estar relacionados.

Realizada la clasificación, se llevó a cabo un nuevo análisis correlacional entre los promedios de los factores que fueron diferentes en el primer análisis (Figura 2) y los errores que fueron clasificados para cada uno de los grados escolares, grupos (PE, CT) y tipos de error (Errores Dictado, Errores Copia, Errores Recuperación). Para el grupo de $1 \mathrm{RO}$ no fueron halladas correlaciones significativas $(p>0.05)$ entre el factor PEG y los errores específicos. Sin embargo, para el grado 2DO, fueron halladas dos tipos de correlación: una correlación negativa entre el factor Cinestésico y los errores específicos en la modalidad de errores de dictado, (grupo CT: $\mathrm{r}=-0.448, \mathrm{p}=0.047$; Figura 5), mostrando que puntajes elevados en la ENIP-S (factor Cinestésico), se relacionaron con un menor número de errores específicos. La tendencia fue exactamente contraria para el grupo PE. Allí, fue encontrada una correlación positiva entre el mismo factor y los errores específicos asociados a él $(\mathrm{r}=0.0510, \mathrm{p}=0.03$; Figura 5), mostrando que puntajes bajos en la ENIP-S se relacionaron con un mayor número de errores específicos. Finalmente, para el grado escolar tercero, las correlaciones fueron realizadas teniendo en cuenta los factores RV y PEA. No fueron halladas correlaciones significativas $(\mathrm{p}>0.05)$, lo que podría sugerir que las correlaciones positivas encontradas en el análisis previo (Figura 4) pudieron obedecer más a los valores de errores específicos no relacionados con los factores analizados. De hecho, las correlaciones entre los factores mencionados y los errores específicos de recuperación, por ejemplo, no pudieron ser 
realizadas dado que en esa modalidad no hubo ningún error en el grado escolar $3 \mathrm{RO}$, es decir, los puntajes fueron cero (0) para todos los participantes.

\section{Figura 5}

Correlación entre Factores Neuropsicológicos y tipo de error II.
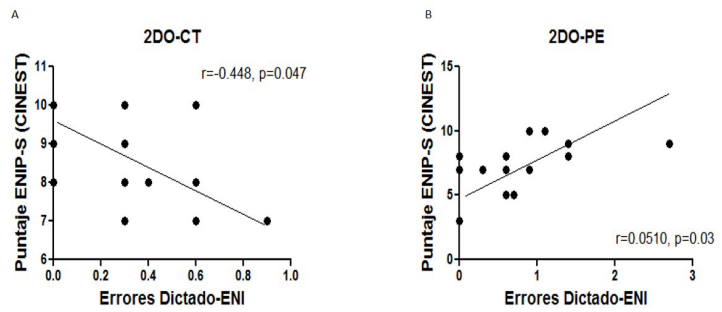

Dentro de cada dimensión de error (dictado Dentro de cada dimensión de error (dictado, copia y recuperación) fueron seleccionados los errores que podrían asociarse específicamente a los factores que mostraron diferencias significativas para cada grado escolar en los primeros análisis (Fig.2). A. Correlación negativa significativa de los puntajes del factor cinético en la ENIP. $\mathrm{S}$ y los errores específicos para este factor en la dimensión "errores de dictado" de la ENI en el grupo CT del grado 2DO. B. Mismo análisis para el grupo PE del grado 2DO. Los valores de $\mathrm{r}$ y $\mathrm{p}$ son mostrados en cada gráfica. El nivel de confianza utilizado fue de $\mathrm{p}<0.05$. Fuente: elaboración propia.

Para finalizar, fue realizado un análisis comparando los grupos CT y PE en cada uno de los errores de dictado asociados al factor cinestésico, con el fin de establecer si dichos errores podrían ser diferentes y cuál podría ser su relación con los resultados previamente obtenidos. Dicho análisis evidencio diferencias significativas, específicamente en sustitución literal en silabas $(p=0.004)$, Sustitución Literal (cinestésica) en No Palabras ( $p=0.033)$, Sustitución Literal $(\mathrm{p}=0.004)$ y Sustitución no ortográfica (cinestésica) $(\mathrm{p}=0.027)$. Los comparaciones entre los errores restantes no mostro ser diferente entre los grupos (ver Tabla Anexo 4).

\section{Discusión}

Las dificultades en la escritura son un tema de constante investigación y debate tanto en la práctica clínica como en la práctica educativa, donde la búsqueda por mejorar en los procesos de enseñanza/aprendizaje es constante. En el presente estudio se evaluó a un grupo de niños y niñas con dificultades en la adquisición de la escritura. Su desempeño fue comparado con un grupo de niños $y$ niñas sin dichas dificultades. Para tal fin, se realizó una evaluación neuropsicológica de tipo "screening", que permite determinar de manera rápida y confiable la presencia o no de alguna dificultad en el desarrollo adecuado de diferentes mecanismos neuropsicológicos (Cisneros, 2009; Sarmiento-Bolaños, Solovieva \& Quintanar, 2013; Solovieva et al., 2013;). Adicionalmente, se evaluó la escritura por medio de tareas específicas, con el fin de buscar correlaciones entre las dificultades de la escritura, manifestadas en los diferentes errores y el estado funcional de desarrollo de los mecanismos neuropsicológicos. Los resultados iniciales, confirmaron una diferencia significativa entre los sujetos control y los experimentales, evidenciando, para los sujetos del grupo CT, un desarrollo funcional adecuado de los diferentes mecanismos neuropsicológicos evaluados. Resultados similares fueron reportados por Canales, Velarde, Meléndez y Lingan, (2013) en su muestra de niños con y sin dificultades de escritura en Perú. En relación con los resultados de este estudio, los puntajes globales del screening, arrojan una diferencia significativa en relación a tres mecanismos específicos: Factor Cinético (Organización secuencial motora), Factor Cinestésico y Factor de Percepción espacial global, todos ellos relacionados con funciones senso-motoras indispensables para la correcta adquisición de la actividad escrita (Mata et al., 2014; Solovieva, Quintanar, \& Lázaro, 2006). Dichos mecanismos han sido señalados en otras investigaciones como predictores de un aprendizaje exitoso de la escritura (Mata et al., 2014; Puranik \& Lonigan, 2014; Puranik, Petscher \& Lonigan, 2014; Solovievaet al., 2013). En cuanto 
María Jimena Sarmiento-Bolaños, Inés Adriana Rojas Ríos, María Jeimy Moreno Carrillo, Alexander Gómez-A.

a la comparación por grupo escolar, los resultados evidenciaron diferencias significativas entre los diferentes grupos que evidencian la complejidad con la que los diferentes mecanismos neuropsicológicos se desarrollan. Así, para el grupo $1 \mathrm{RO}$, el factor neuropsicológico con menor desarrollo funcional fue Percepción Espacial Global. Este resultado es acorde con la literatura dado que este tipo de percepción se relaciona con zonas terciarias posteriores derechas (TPO), las cuales maduran de forma más tardía junto con las zonas terciarias anteriores (frontales). Las primeras (zonas TPO), estarían concluyendo su desarrollo a la edad de 7 años mientras que para las segundas (zonas frontales), el desarrollo se extiende hasta la adolescencia (Flores-Lázaro, 2010; Luria, 1969). El escaso desarrollo de este mecanismo o factor, se evidencia en la dificultad para percibir de forma global los diferentes estímulos, es decir, una percepción basada en elementos de forma y no de detalle, en ubicación general de características topológicas como la distribución de los elementos en el espacio gráfico, la proporción y la formación del esquema espacial de la palabra en general (Akhutina, 2000; Lázaro \& Ostrosky-Solís, 2012). De esta manera, el grupo 1 RO presenta fallas de este tipo que podrían subyacer a las dificultades en la adquisición de la escritura, siendo éste uno de los eslabones claves para tener en cuenta durante el proceso de enseñanza de la misma. Adicionalmente, es importante destacar la relación de otras actividades, (cada vez más ausentes en pre-escolares y escolares menores), con el desarrollo de este mecanismo de Percepción Espacial Global, tales como la actividad gráfica (especialmente dirigida), la cual ha mostrado una fuerte influencia en el desarrollo de las capacidades relacionadas con este factor y con la escritura como actividad simbólica (Mata et al., 2014; Sarmiento-Bolaños \& Castellanos, 2015) y la actividad física (Carey \& Gelman, 2014; Diamond \& Gilbert, 1989).

Por su parte, el grupo escolar 2DO presentó dificultades en el desarrollo del mecanismo Cinestésico, relacionado con el funcionamiento de zonas parietales que, en el caso específico de la escritura, aportaría para la correcta identificación de sonidos de acuerdo a su base cinestésica, es decir, al punto y modo de articulación de los diferentes sonidos que, posteriormente, serán relacionados con una grafía específica. Adicionalmente, este mecanismo aportaría la base cinestésica-sensorial aferente para el movimiento implicado en la escritura, su fluidez y precisión (Luria, 2005). En la práctica clínica, este tipo de dificultades son más comúnmente relacionadas con la memoria visual o con procesos atencionales y solo una evaluación neuropsicológica precisa muestra este tipo de dificultades. De acuerdo a la lógica del desarrollo de los diferentes mecanismos neuropsicológicos, este resultado evidencia que los niños de este grado escolar presentan un desarrollo funcional inadecuado de este factor. Estos resultados no coinciden con lo reportado por Cisneros (2009), quien evalúo a niños de segundo a sexto grado de escuela primaria en México, reportando que el factor cinestésico mostró un alto grado de desarrollo en los niños del grado escolar 2DO.

La diferencia con nuestros resultados, podría obedecer al hecho de que en México, la enseñanza formal de la escritura comienza en el primer año de la escuela primaria, lo que proporcionaría una mayor oportunidad para trabajar en el desarrollo de los pre-requisitos de la escritura, como sería el caso del factor cinestésico. En la muestra evaluada para este estudio, el proceso de lecto-escritura comienza formalmente en el tercer año de pre-escolar. Por último, el grado escolar $3 \mathrm{RO}$, presenta diferencias significativas en el desarrollo de los factores Retención visual y Percepción Espacial Analítica que, siguiendo con el curso del desarrollo, tendrían sentido. En el caso de la percepción espacial de tipo analítica, relacionada con el desarrollo de zonas terciarias posteriores (TPO), (en este caso izquierdas), se relaciona con la capacidad para percibir diferentes estímulos, basada no en la forma general o global como en etapas iniciales del desarrollo, sino en el aspecto particular y analítico de los detalles. Asimismo, las zonas TPO participan en el reconocimiento de características topológicas específicas como la diferenciación de derechaizquierda y aquellas características más básicas 
(arriba, abajo, adelante, atrás, en medio), esta vez simbolizadas por medio del lenguaje, en lo que conocemos como preposiciones. Estas funciones del mecanismo de percepción espacial analítica, permiten la diferenciación de grafías (letras) similares espacialmente (pbdq/7f/96), así como de números y otros elementos gráficos con especificidad en sus características espaciales (Eslava-Cobos \& Mejía, 2008). Este tipo de alteración ha sido reportada en otros estudios como dificultades de la decodificación grafémica (Geraldi, Haase, Oliveira, Luiz, Ferreira \& Figuereido, 2011).

Sin embargo, este término probablemente no sería el indicado para describir la dificultad en sí, dado que engloba muchas otras más dificultades además del análisis espacial analítico como el análisis visual y fonémico. Un elemento importante en esta discusión, es la presencia, de manera constante, en los tres grados escolares evaluados, de la alteración (si bien no significativa estadísticamente, sí cercana al nivel de significancia) del factor Cinético (ver Figura 2). Este mecanismo ya había sido mencionado dentro de la comparación general de los grupos, como uno de los factores alterados en el grupo PE, relacionándolo como uno de los principales responsables del proceso de interiorización o mecanización de la escritura. Estos resultados fueron igualmente reportados por Cisneros (2009) para los grados segundo y tercero de primaria. En términos de desarrollo, el factor Cinético, relacionado con zonas frontales posteriores pre-motoras y sus conexiones con los ganglios basales principalmente, constituyen la base para lo que se conoce como memoria de procedimiento, participando en etapas tardías del aprendizaje de la escritura en el aspecto mecánico, aunque también en etapas iniciales, en el aspecto de la organización secuencial (Akhutina, 2000). En otras investigaciones (Canales et al., 2013), el factor Cinético ha sido reportado como base de la escritura productiva, específicamente con la velocidad de la escritura, posiblemente por su relación con la organización secuencial motora interiorizada.

En cuanto a las dificultades específicas de la escritura, manifestadas en los diferentes errores cometidos por los participantes, subdivididos en errores de dictado, copia y recuperación, un primer análisis, comparando la ejecución total del grupo control y experimental por grados, no mostró diferencias en $1 \mathrm{RO}$, pero sí en los otros grados escolares. Una primera explicación para este hallazgo se puede relacionar con el proceso de aprendizaje de la escritura. Dado que el grupo $\mathrm{CT}$ y el PE en el grado $1 \mathrm{RO}$ se encuentran en una etapa inicial de dicho aprendizaje, sería de esperar ejecuciones similares y que las pocas diferencias no sean tan evidentes, por lo menos en el aspecto cuantitativo. Si bien el grupo PE mostró un menor grado de desarrollo del factor Espacial Global, las tareas de escritura aplicadas no valoran cuantitativamente aspectos íntimamente relacionados con este factor, (descritos previamente), aunque cualitativamente estas diferencias sean evidentes. En el caso del grado 2DO, se hallaron diferencias en dictado, donde los niños y niñas del grupo PE cometieron más errores. Este resultado puede asociarse al obtenido en la evaluación neuropsicológica, donde fueron evidenciadas fallas específicas en el factor Cinestésico del grupo PE, lo cual, adicionalmente, encontró soporte en las correlaciones entre el factor cinestésico y los errores de dictado, al ser halladas correlaciones negativas para el grupo CT y positivas para el grupo PE. Todo esto permite concluir que las fallas en dictado de este grupo se deben a un menor desarrollo del factor cinestésico, el cual, como se mencionó anteriormente, proporciona la base sensorial (a nivel del aparato fonoarticulatorio) para el análisis de los sonidos percibidos y su correspondiente transformación al código escrito. Por último, los resultados del grado escolar3RO mostraron diferencias entre los grupos CT y PE en las tareas de dictado $y$, de forma marginal, en copia y recuperación. Este resultado podría ser interpretado como incoherente con la lógica del desarrollo y automatización de la actividad escrita, sin embargo, estudios recientes sugieren que en la escritura automatizada, las funciones de más alto orden como el sentido, la planeación y la ideación, consumen la mayor cantidad de recursos (Akhutina, 2002; Moats, 2005), se 
aumenta la fluencia al escribir (Kim, Al Otaiba, Puranik, Folsom, Greulich \& Wagner, 2011) y por tanto, se incrementa la posibilidad de cometer errores en la producción escrita. Con respecto a esto y basados en la ausencia de correlaciones entre estos errores y los factores neuropsicológicos afectados para el grado 3RO, podemos inferir, (basados nuevamente en la lógica del desarrollo neuropsicológico y en la conformación del sistema funcional de la escritura), que en este grado escolar los errores de escritura responden a una inicial participación del mecanismo o factor neuropsicológico de Regulación y Control en la etapa de verificación de la actividad y comparación con el objetivo inicial y, además, que la participación de dicho factor en actividades complejas como la escritura, requiere de una enseñanza particular.

Por último, los resultados mostraron que ciertos tipos de error específicos para tareas de dictado como el error de sustitución literal en sílabas, no palabras y oraciones, resultaron ser significativos al relacionarse con el factor neuropsicológico cinestésico. Lo anterior da soporte a los diferentes resultados obtenidos en este estudio en cuanto evidencia la falla del factor cinestésico, especialmente en tareas de dictado, donde fue evidente la presencia de diferentes sustituciones del tipo literal cinestésicas, es decir, de letras similares por sus característica articulatorias (punto y modo de articulación), lo que puede constituirse como un punto de referencia importante durante el diseño de intervenciones y prácticas pedagógicas dirigidas a la enseñanza de la lecto-escritura.

\section{Conclusiones}

El presente estudio permite obtener las siguientes conclusiones con respecto a la población y proceso estudiados:

1. Las alteraciones neuropsicológicas y de la escritura encontradas en la población estudiada coinciden con la lógica del desarrollo neuropsicológico en cuanto involucran, inicialmente, mecanismos cerebrales posteriores (factores espacial y cinestésico) responsables de las funciones sensoriales básicas necesarias para la adquisición de la escritura y posteriormente, mecanismos cerebrales anteriores (regulación y control) responsables del mantenimiento y verificación de dicha actividad.

2. El factor o mecanismo neuropsicológico Cinestésico y su desarrollo adecuado o no, puede ser considerado como un predictor de la adquisición de la escritura y la tarea de dictado puede ser considerada como una tarea sensible para el reconocimiento de las dificultades en dicho factor y en la escritura.

3. De acuerdo a lo observado en esta población, los métodos de enseñanza de la lectura deben incluir en su estructura actividades previas que apoyen el desarrollo de los factores aquí relacionados con la adquisición de la escritura en sus etapas iniciales (Factor espacial global y analítico, cinestésico y cinético) y tardías (regulación y control).

\section{Referencias}

Akhutina, T. (2002). Diagnóstico y corrección de la escritura. Revista española de Neuropsicología, 4(2-3), 236-261.

Bravo-Valdivieso, L., Milicic-Müller, N., Cuadro, A., Mejía, L. \& Eslava, J. (2015). Trastornos del aprendizaje: investigaciones psicológicas y psicopedagógicas en diversos países de Sudamérica. Ciencias Psicológicas, 3(2), 203-218.

Carey, S., \& Gelman, R. (2014). The epigenesis of mind: Essays on biology and cognition. New York: PsychologyPress.

Canales, R., Velarde, E., Meléndez, C., \& Lingán, S. (2013). Factores neuropsicológicos y procesos cognitivos en niños con retraso en la escritura y sin retraso en la escritura: Propósitos y Representaciones. Revista de Educación y Psicología de la USIL, 1(2), 11-29. 
Cisneros, N. (2009). Características neuropsicológicas del proceso de la escritura en niños con problemas en el aprendizaje de 7 a 12 años. Maestría en diagnóstico y rehabilitación neuropsicológica, Benemérita Universidad Autónoma de Puebla, México.

Diamond, A. \& Gilbert, J. (1989). Development as progressive inhibitory control of action: Retrieval of a contiguous object. Cognitive Development, 4(3), 223-249.

Eslava-Cobos J. \& Mejía L. (2008). Aproximación Neurofisiológica: Evaluación de los Trastornos del Aprendizaje. En Eslava-Cobos J., Mejía L., Quintanar L. \& Solovieva Y. (Eds.). Los Trastornos del Aprendizaje: Perspectivas Neuropsicológicas. Bogotá, Editorial Magisterio.

Flores-Lázaro, J. (2010). Evaluación Neuropsicológica del Daño Frontal: Sistematización y Aplicación del Enfoque de A.R. Luria. Revista Neuropsicología, Neuropsiquiatría y Neurociencias. 10(2), 57-68.

García, J., Madrazo, M. \& Viñals, A. (2002). Alteraciones del procesamiento en la escritura: la disgrafía superficial. Revista Española de Neuropsicología (4), 283-300.

Geraldi Haase, V., de Oliveira Ferreira, F., Freitas, L., Ferreira Ramos, P. \& Figueiredo Silva, N. (2011). Habilidades de decodificação grafêmica e fonêmica: um estudo populacional do desempenho de escolares. Estudos da Língua (gem). 9 (2) 91-105.

González, M., Martín, I. \& Delgado, M. (2011). Intervención temprana de la lectoescritura en sujetos con dificultades de aprendizaje. Revista Latinoamericana de Psicología. 43(1), 35-44.

Kim, Y. S., Al Otaiba, S., Puranik, C., Folsom, J. S., Greulich, L., \& Wagner, R. K. (2011). Componential skills of beginning writing: An exploratory study. Learning and Individual Differences, 21(5), 517-525.

Lázaro, J. C. F. \& Ostrosky-Solís, F. (2012). Desarrollo neuropsicológico de lóbulos frontales y funciones ejecutivas. Editorial $\mathrm{El}$ Manual Moderno

Loredo, D. (2008). Caracterización neuropsicológica de una población infantil urbana. Maestría en diagnóstico y rehabilitación neuropsicológica, Benemérita Universidad Autónoma de Puebla, México.

Londoño-Muñoz, N., Jiménez-Jiménez, S., González-Alexander, D. C. \& Solovieva, Y. (2016). Análisis de los errores en la lectura y en el lenguaje escrito en niños de Educación Primaria. Ocnos: Revista de estudios sobre lectura, 15(1), 97-113

Luria, A.R. (1950). Ensayos sobre la psicofisiología de la escritura. Moscú, Academia de Ciencias Pedagógicas de la Federación Rusa.

Luria, A.R. (1969). Exploración de las funciones corticales superiores, Volumen III. Barcelona: Ediciones Martínez Roca.

Luria, A. R., (2005). Las funciones corticales superiores del hombre. México: Fontamara.

Mata, A., Solovieva, Y., Quintanar, L. \& Soto, F. (2014). Utilidad del dibujo para superar problemas en la escritura: estudio de un caso. Revista Chilena de Neuropsicología, 9 (2), 54-60.

Moats, L. C. (Winter 2005). How spelling supports reading: And why it is more regular and predictable than you think. American Educator, 6(12-22), 42-43.

Puranik, C.S. \& Lonigan, C.J. (2014). Emergent Writing in Preschoolers: Preliminary Evidence for a Theoretical Framework. Reading Research Quarterly. 49 (4) 453-467.

Puranik, C. S., Petscher, Y. \& Lonigan, C. J. (2014). Learning to write letters: Examination of student and letter factors. Journal of experimental childpsychology, 128, 152-170.

Quintanar, L., \& Solovieva, Y. (2002). Análisis neuropsicológico de las alteraciones del lenguaje. Revista de Psicología General y Aplicada. 55(1), 67-87.

Rivera, C., Rosas, R., Ceric, F., Tenorio, M., Mourgues, C. \& Hurtado, E. (2011). Análisis preliminar de la validez 
María Jimena Sarmiento-Bolaños, Inés Adriana Rojas Ríos, María Jeimy Moreno Carrillo, Alexander Gómez-A.

interna y convergente de la Evaluación Neuropsicológica Infantil Breve con pruebas de dominio específico Batería UC. XII Congreso de la Sociedad Latinoamericana de Neuropsicología (SLAN), Santiago de Chile.

Rojas, L. Q., \& Solovieva, Y. (2005). Análisis neuropsicológico de los problemas en el aprendizaje escolar. Revista Internacional del Magisterio (Colombia), 15, 26-30.

Rosselli, M., Matute, E., Ardila, A., Botero, V., Tangarife, G., Echeverría, S., ... Ocampo, P. (2004). Evaluación Neuropsicológica Infantil (ENI) una batería para la evaluación de niños entre 5 y 16 años de edad. Estudio normativo colombiano. Revista de Neurología. 38(8), 720-731.

Sarmiento-Bolaños, M. J. \& Castellanos, J. (2015). Formación de la actividad gráfica en pre-escolares: aportes desde la neuropsicología histórico-cultural. Poiésis, 9 (15) 8-23.

Sarmiento-Bolaños, M. J., Solovieva, Y. \& Quintanar, L. (2013). Perfil Neuropsicológico en niños de 5 a 7 años de edad con traumatismo craneoencefálico. Revista Neuropsicología Latinoamericana, 5(4) 39-52.

Solovieva, Y., Loredo, D., Quintanar, L. \& Lázaro, E. (2013). Caracterización neuropsicológica de una población infantil urbana a través de la Evaluación Neuropsicológica Infantil Puebla-Sevilla. Pensamiento psicológico, 11 (1), 83-98.

Solovieva, Y., Quintanar Rojas, L. \& Lázaro, E. (2006). Efectos socioculturales sobre el desarrollo Psicológico y neuropsicológico en niños preescolares. Cuadernos Hispanoamericanos de Psicología,6(1), 9-20.

Solovieva, Yu., Quintanar, L. León-Carrión J. (2007). Evaluación neuropsicológica infantil "Puebla-Sevilla" Screening Neuropsicológico Clínico. México, Benemérita Universidad Autónoma de Puebla.

Vigotsky, L. S. (1979). El desarrollo de los procesos psicológicos superiores (pp. 159-178). M. Cole (Ed.). Barcelona: Crítica.
Yañez G. (2008). Aproximación cognoscitiva: evaluación de los trastornos del aprendizaje. En Eslava-Cobos J., Mejía L., Quintanar L. \& Solovieva, Y. (Eds.). Los trastornos de aprendizaje: perspectivas neuropsicológicas. Textos de neuropsicología Latinoamericana. Tomo 1. Colombia, Magisterio: 309-336.

\section{Anexo}

\section{Tabla S1}

Mecanismos neuropsicológicos involucrados en las acciones que componen la escritura

\begin{tabular}{|c|c|c|}
\hline FUNCIÓN & FACTOR & ESLABÓN \\
\hline Análisis de los elementos & Perceptivo visual & Imagen visual de la letra \\
\hline Diferenciación de letras similares & Espacial & Imagen visuo-espacial \\
\hline $\begin{array}{l}\text { a) Esquema de movimientos } \\
\text { de acuerdo a la imagen } \\
\text { b) Movimientos finos, pasos } \\
\text { fluentes de un elemento al } \\
\text { otro. }\end{array}$ & $\begin{array}{l}\text { a) Cinestésico } \\
\text { b) Cinético }\end{array}$ & Ejecución de la escritura \\
\hline $\begin{array}{l}\text { Objetivo, elección del programa de } \\
\text { combinaciones de letras y palabras, } \\
\text { control de la comprensión del } \\
\text { sentido y de los signos de } \\
\text { puntuación. }\end{array}$ & Regulación voluntaria & Intención \\
\hline $\begin{array}{l}\text { Diferenciación de articulemas } \\
\text { cercanos }\end{array}$ & Cinestésico & $\begin{array}{l}\text { Correlación entre sonido y } \\
\text { letra a traveśs de la } \\
\text { pronunciación }\end{array}$ \\
\hline $\begin{array}{l}\text { Diferenciación de fonemas (vocal- } \\
\text { consonante, sordos, sonoros, etc.) }\end{array}$ & Fonemático & Percepción de sonidos \\
\hline Material para la escritura & $\begin{array}{l}\text { Modal específico } \\
\text { (auditivo) }\end{array}$ & Memoria audio-verbal \\
\hline $\begin{array}{l}\text { Rapidez, tamaño, intervalos, } \\
\text { página. }\end{array}$ & Neurodinámico & Estabilidad de la escritura \\
\hline
\end{tabular}

\section{Tabla S2}

Criterios de inclusión y exclusión de los niños y niñas evaluados

\begin{tabular}{ll}
\hline \multicolumn{1}{c}{ Criterios exclusión } & \multicolumn{1}{c}{ Criterios inclusión } \\
\hline Presentar antecedentes neurológicos & $\begin{array}{l}\text { Pertenecer a la institución educativa } \\
\text { seleccionada en el municipio de Cota } \\
\text { (Cundinamarca) }\end{array}$ \\
\hline $\begin{array}{l}\text { Presentar un déficit sensorial (Ceguera, } \\
\text { Sordera, Mudez) }\end{array}$ & $\begin{array}{l}\text { Estar cursando primero, segundo o } \\
\text { tercero de básica primaria }\end{array}$ \\
\hline $\begin{array}{l}\text { Presentar alguna alteración en el } \\
\text { estado del ánimo }\end{array}$ & \begin{tabular}{l} 
Tener entre 7 y 10 años \\
\hline
\end{tabular} \\
\hline $\begin{array}{l}\text { Tener reporte del docente de Inglés de } \\
\text { presentar un bajo rendimiento en la } \\
\text { escritura }\end{array}$ \\
\hline $\begin{array}{l}\text { Tener reporte del docente de Español } \\
\text { de presentar un bajo rendimiento en la } \\
\text { escritura }\end{array}$ \\
\hline
\end{tabular}




\section{Tabla S3}

Caracterización demográfica de la muestra

\begin{tabular}{cccl}
\hline 3RO & 2DO & 1RO & Variable \\
\hline 12 & & & Genero \\
\hline 8 & 14 & 10 & Hombres \\
\hline 9,1 & 6 & 10 & Mujeres \\
\hline Asignación por grupo & & & Edad \\
\hline 10 & 18 & 7,1 & Media (años) \\
\hline 9 & $2\left(20^{*}\right)$ & 11 & Experimentales \\
\hline
\end{tabular}

* El grupo control para el grado escolar 2DO se compuso de la totalidad de sujetos asignado al grupo control para el estudio (ver resultados).

\section{Tabla $\mathbf{S 4}$}

Tipos de errores en las tareas de dictado prueba ENI

\begin{tabular}{|c|c|c|c|c|c|c|c|}
\hline \multirow{3}{*}{$\begin{array}{l}\text { Tipos de errores en } \\
\text { tareas de dictado }\end{array}$} & \multicolumn{6}{|c|}{ Descriptivos } & \multirow{3}{*}{$\begin{array}{c}\text { Resultados } \\
\text { t-Student } \\
P=<0,05\end{array}$} \\
\hline & \multicolumn{2}{|c|}{$\mathbf{N}$} & \multicolumn{2}{|c|}{ Media } & \multicolumn{2}{|c|}{$\begin{array}{l}\text { Desviación } \\
\text { Estándar }\end{array}$} & \\
\hline & CT & PE & CT & PE & CT & $P E$ & \\
\hline $\begin{array}{l}\text { Sustitución Literal en } \\
\text { Silabas }\end{array}$ & 20 & 18 & 0,20 & 1,000 & 0,410 & 1,085 & $0,0041^{*}$ \\
\hline $\begin{array}{l}\text { Sustitución Literal } \\
\text { (espacial) en palabras }\end{array}$ & 20 & 18 & 0,05 & 0,278 & 0,224 & 0,669 & 0,1592 \\
\hline $\begin{array}{l}\text { Sustitución Literal } \\
\text { (cinestésica) en Palabras }\end{array}$ & 20 & 18 & 0,15 & 0,167 & 0,366 & 0,515 & 0,9084 \\
\hline $\begin{array}{l}\text { Sustitución Literal } \\
\text { (cinestésica) en No } \\
\text { Palabras }\end{array}$ & 20 & 18 & 0,35 & 0,889 & 0,587 & 0,900 & $0,0338^{*}$ \\
\hline $\begin{array}{l}\text { Sustitución Literal } \\
\text { (cinestésica) en } \\
\text { Oraciones }\end{array}$ & 20 & 18 & 0,20 & 0,556 & 0,523 & 1,199 & 0,2357 \\
\hline Sustitución Literal & 20 & 18 & 0,20 & 1,000 & 0,410 & 1,085 & $0,0041^{*}$ \\
\hline $\begin{array}{l}\text { Sustitución no ortográfica } \\
\text { (cinestésica) }\end{array}$ & 20 & 18 & 0,70 & 1,778 & 0,801 & 1,927 & $0,0278^{*}$ \\
\hline
\end{tabular}

\section{Notas}

* Artículo de investigación. 